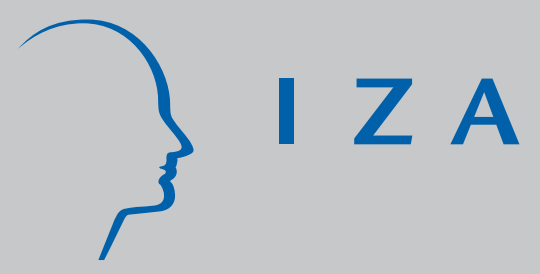

IZA DP No. 3835

Promotions and Incentives:

The Case of Multi-Stage Elimination Tournaments

Steffen Altmann

Armin Falk

Matthias Wibral

November 2008 


\title{
Promotions and Incentives: The Case of Multi-Stage Elimination Tournaments
}

\author{
Steffen Altmann \\ University of Bonn and IZA
}

Armin Falk

University of Bonn and IZA

Matthias Wibral

University of Bonn and IZA

\section{Discussion Paper No. 3835 \\ November 2008}

** revised and extended version forthcoming in: Journal of Labor Economics **

\author{
IZA \\ P.O. Box 7240 \\ 53072 Bonn \\ Germany \\ Phone: +49-228-3894-0 \\ Fax: +49-228-3894-180 \\ E-mail: iza@iza.org
}

Any opinions expressed here are those of the author(s) and not those of IZA. Research published in this series may include views on policy, but the institute itself takes no institutional policy positions.

The Institute for the Study of Labor (IZA) in Bonn is a local and virtual international research center and a place of communication between science, politics and business. IZA is an independent nonprofit organization supported by Deutsche Post World Net. The center is associated with the University of Bonn and offers a stimulating research environment through its international network, workshops and conferences, data service, project support, research visits and doctoral program. IZA engages in (i) original and internationally competitive research in all fields of labor economics, (ii) development of policy concepts, and (iii) dissemination of research results and concepts to the interested public.

IZA Discussion Papers often represent preliminary work and are circulated to encourage discussion. Citation of such a paper should account for its provisional character. A revised version may be available directly from the author. 


\section{ABSTRACT}

\section{Promotions and Incentives: The Case of Multi-Stage Elimination Tournaments ${ }^{*}$}

Promotion tournaments play an important role for the provision of incentives in firms. In this paper, we extend research on single-stage rank-order tournaments and analyze behavior in multi-stage elimination tournaments. The main treatment of our laboratory experiment is a two-stage tournament in which equilibrium efforts are the same in both stages. We compare this treatment to a strategically equivalent one-stage tournament and to another two-stage tournament with a more convex wage structure. Confirming previous findings average effort in our one-stage treatment is close to Nash equilibrium. In contrast, subjects in our main treatment provide excess effort in the first stage both with respect to Nash predictions and compared to the equivalent one-stage tournament. The results for the more convex twostage tournament show that excess effort in the first stage is a robust finding and that subjects react only weakly to differences in the wage structure.

JEL Classification: M51, M52, J33, C92

Keywords: personnel economics, tournament, incentives, laboratory experiment

Corresponding author:

Matthias Wibral

Institute for Empirical Research in Economics

University of Bonn

Adenauerallee 24-42

53113 Bonn

Germany

E-mail: wibral@uni-bonn.de

\footnotetext{
Financial support from the Deutsche Forschungsgesellschaft through SFB/TR 15 is gratefully acknowledged. We thank Johannes Abeler, Michael Bognanno, Florian Ederer, Ernst Fehr, Uri Gneezy, Sotiris Georganas, Lars Koch, Matthias Kräkel, Felix Marklein, Robert Oxoby, Bruce Weinberg, and Georg Weizsäcker for helpful discussions. Valuable comments were also received from seminar and conference participants in Bonn, Barcelona, Budapest, Cologne, Malaga, Munich, New Orleans, Nottingham, Nuremberg, Raleigh, San Diego, Tübingen, and Warwick. Part of this research was conducted while two of the authors were visiting the Institute for Empirical Research in Economics at the University of Zurich. The authors thank the institute and especially Christian Ewerhart for their hospitality, encouraging discussions, and financial support.
} 


\section{Introduction}

Promotions play an important role for the provision of incentives in firms and other hierarchical organizations. According to Lazear and Gibbs (2008), they are probably the most important source of extrinsic motivation for middle managers in most firms. The prospect of being promoted to a better-paid job creates incentives to work hard, even if current income is not tied to performance. In virtually all firms those who get promoted compete again for subsequent promotions: in many companies, there are up to a dozen hierarchical levels between the CEO and entry-level management (Belzil and Bognanno 2008, Lazear and Gibbs 2008). The prevalence of internal labor markets is also underlined by the fact that hiring is concentrated on lower levels of the hierarchy and that positions on higher ranks are filled primarily through promotion (Baker et al. 1994, Bognanno 2001). In a seminal contribution, Rosen (1986) has modelled the competition for promotion in such hierarchies as a multi-stage elimination tournament where in each stage fewer agents are selected for the next step of the career ladder. Incentives generated in such tournaments depend on two important components of the organizational structure: the immediate wage increase for an agent who gets promoted and the option value of competing in further stages of the tournament, i.e., having the chance to earn even higher wages.

Although the importance of multi-stage elimination tournaments is undisputed, stringent empirical tests of their incentive effects are scarce. In this paper, we provide a step towards closing this gap with the help of a controlled laboratory experiment. Our main questions are: do people take future stages of a tournament into account when deciding on current effort? Are multi-stage elimination setups behaviorally different from simple one-stage tournaments? How does the wage structure in multi-stage tournaments affect the provision of effort?

We study these questions by comparing three treatments. Our main treatment is a two-stage tournament $(T S)$ in which four subjects compete for being promoted to the second stage. Promotion depends on subjects' output which is a function of costly 
effort and an individual noise term. The two subjects with the lowest output levels in the first stage are eliminated from further competition and receive a low wage. The two subjects with the highest output levels in the first stage are promoted, i.e., they are allowed to take part in the second stage where they compete against each other again. The subject with the highest second-stage output receives a high wage, whereas the other finalist is paid an intermediate wage. Parameters in this treatment are chosen to make the tournament incentive maintaining in the sense that equilibrium effort is identical in both stages (Rosen 1986).

We compare this treatment to a one-stage tournament $(O S)$ in which four subjects compete once for two top positions. Wages for the promoted subjects are chosen such that the one-stage tournament is strategically equivalent to the first stage of $T S$. This means that promoted subjects in $O S$ earn the sum of the intermediate wage in $T S$ and the monetary equivalent of the second-stage option value in that tournament, implying that equilibrium effort is the same in both treatments. Comparing $O S$ and $T S$ thus allows testing whether strategic equivalence translates into behavioral equivalence. Our third treatment $(T S C)$ is identical to the $T S$ treatment with the exception that the wage structure is more convex, i.e., the intermediate wage is smaller in TSC. It is designed to study how subjects react to differences in the wage structure. In particular, we can test whether subjects - as predicted by theory - exert lower efforts in the first and higher efforts in the second stage under the more convex wage structure of TSC compared to $T S$.

Our findings can be summarized as follows: First, average behavior is remarkably close to the predictions of tournament theory in our one-stage treatment. This parallels findings of previous experiments on symmetric one-stage tournaments (e.g., Bull et al. 1987, Orrison et al. 2004). Second, behavior in the TS treatment indicates that subjects take the option value of future promotion possibilities into account when deciding on their work effort in multi-stage tournaments. Third, we also observe important departures from theoretical predictions in the TS treatment. Behavior in the first stage of TS differs strongly both from the one-stage treatment and from theoretical predictions. 
Subjects exert significantly higher efforts in the first stage of the two-stage tournament. Fourth, the results of the TSC treatment confirm the occurrence of excess effort provision in the first stage of the tournament. Subjects react only weakly to the change in the wage structure, implying that first-stage excess effort is even higher in TSC. Finally, the $T S$ treatment is incentive maintaining in the sense that efforts in the second stage are not significantly different from first-stage efforts.

Most promotion tournaments that we observe in firms and other hierarchical organizations have multiple stages. Our results indicate that the mechanisms of incentive provision in multi-stage tournaments largely operate as suggested by theory. People do not only respond to differences in prizes, or wages, but are also motivated by the option value generated by future promotion possibilities. Our paper also provides insights with regard to the question whether one-stage tournaments are behaviorally equivalent to multi-stage designs. Adding one or more stages seems to make a fundamental difference, as people tend to exert excess effort in early stages of the tournament. This shows that one cannot necessarily draw inferences from simple one-stage setups to more complex tournaments.

The finding that people tend to exert excess effort in early stages of a multistage competition also has interesting organizational implications. If - as our findings suggest - multi-stage competitions provoke excess effort exertion by employees, this may offer a possible explanation why firms rely heavily on promotion based incentive schemes even if more direct means of performance assessment and compensation are available. Excess effort makes a promotion tournament "cheaper" for principals compared to other incentive schemes such as piece rates or bonus contracts. Moreover, excess effort influences the "optimal architecture" of promotion tournaments and other contests (see Moldovanu and Sela 2006).

Our paper complements previous studies which have used field data from executive compensation, sports, or agricultural production to evaluate predictions of tournament theory (e.g., Ehrenberg and Bognanno 1990, Knoeber and Thurman 1994, Bognanno 2001). It has been found that many implications of tournament theory are consistent 
with empirical observations: for instance, higher prizes tend to increase performance (Ehrenberg and Bognanno 1990), wage profiles in the field are convexly increasing (Eriksson 1999), and winner prizes increase with the number of competitors (Bognanno 2001). An advantage of testing tournament theory with laboratory experiments is the possibility to directly test pure incentive effects of tournaments. For instance, one can measure agents' behavioral reactions to changes in the wage structure without being concerned that the composition of the workforce might change due to the modified incentive scheme (Lazear 2000, Dohmen and Falk 2006). Experimental data also allows to rule out influences of unobservable variables that might affect actual promotion decisions in the field, like agents' soft skills or supervisor favoritism.

A number of previous papers have studied various aspects of tournaments in experiments, such as the effects of different prize spreads (Bull et al. 1987, Harbring and Irlenbusch 2003), sabotage activities (Falk et al. 2008, Harbring and Irlenbusch 2008) or asymmetries in promotion chances (Schotter and Weigelt 1992). In contrast to our paper this literature concentrates on one-stage tournaments. In view of our findings it is not clear to what extent these previous findings translate to multi-stage setups. For instance, more competitive behavior (excess effort) in early stages might also lead to an increase in sabotage activities in multi-stage tournaments.

The remainder of the paper is organized as follows: the next section presents a simple model of multi-stage elimination tournaments on which our experiment is based. Section 3 discusses our experimental design and derives hypotheses. Section 4 shows the main results and section 5 concludes. 


\section{A simple model of multi-stage elimination tournaments}

We consider a simple elimination tournament in which four identical agents compete for promotion. ${ }^{1}$ The promotion decision depends on relative output produced by the agents. Competition consists of two stages: in the first stage, all four agents compete against each other. The two agents with the lowest output levels in that stage receive a wage $w_{\text {low }}$ and are eliminated from further competition. The two agents with the highest output levels in the first stage are promoted, i.e., they are allowed to take part in the second stage (or "final") where they compete against each other again. The agent who produces more output in the second stage receives a wage $w_{h i g h}$, whereas the other finalist gets an intermediate wage $w_{m e d}$. Note that the decision of who receives $w_{\text {high }}$ or $w_{\text {med }}$ does not depend on the first-stage output of the finalists.

This two-stage elimination tournament can be modeled as follows. In the first stage of the tournament four agents $i=1,2,3,4$ compete against each other. Agents who participate in stage $k \in\{1,2\}$ individually produce output $y_{i, k}$ according to the production function

$$
y_{i, k}=e_{i, k}+\epsilon_{i, k}
$$

where $e_{i, k}$ denotes the effort level that agent $i$ exerts in stage $k . \epsilon_{i, k}$ is a random shock faced by agent $i$ in stage $k$. Shocks are assumed to be drawn independently for each agent in each stage. For simplicity, we assume that $\epsilon_{i, k}$ is uniformly distributed on the interval $[-q, q]{ }^{2}$ Agent $i$ 's output in stage $k$ does not depend on previous effort or output and the production technology is identical for all agents $i$ in all stages $k$. Agents

\footnotetext{
${ }^{1}$ Most of the assumptions below follow the classic (one-stage) tournament model introduced by Lazear and Rosen (1981).

${ }^{2}$ Virtually all tournament experiments use the uniform distribution, primarily because its concept is easy to understand for experimental subjects. The predictions of the model, however, can be generalized to other distributions of shocks. Lazear and Rosen (1981) discuss which conditions have to be fulfilled for the existence of an equilibrium in pure strategies.
} 
bear the cost of effort exertion. We assume the cost function to be of the following form:

$$
C\left(e_{i, 1}, e_{i, 2}\right)=\frac{e_{i, 1}^{2}}{c}+\frac{e_{i, 2}^{2}}{c}
$$

Note that this specification implies separability of costs across stages, i.e., in line with Rosen (1986) there is also no carry-over of costs between stages. Furthermore, we assume that agents are identical and risk-neutral with utility functions which are additively separable in wages and effort costs

$$
U_{i}\left(w, e_{i, 1}, e_{i, 2}\right)=w-C\left(e_{i, 1}, e_{i, 2}\right)
$$

For the derivation of equilibrium predictions we restrict our attention to the set of symmetric subgame perfect Nash equilibria. The two-stage tournament can be solved by backward induction. Because (i) the decision who wins the second stage solely depends on the output of the finalists in this stage, (ii) there is no cost carry-over between stages and (iii) the random terms are independently distributed both across stages and agents, the final of our two-stage tournament is equivalent to a simple onestage tournament in which two participants compete for a promotion. Given that agent $i$ has reached the second stage where two agents compete for one winner prize $w_{h i g h}$ and one loser prize $w_{m e d}$, he chooses stage-two effort $e_{i, 2}$ in order to maximize an expected utility function of the following form: ${ }^{3}$

$$
E U_{i}\left(w_{h i g h}, w_{m e d}, e_{i, 2}, e_{j, 2}\right)=\pi\left(y_{i, 2}>y_{j, 2}\right) w_{h i g h}+\left[1-\pi\left(y_{i, 2}>y_{j, 2}\right)\right] w_{m e d}-C\left(e_{i, 1}, e_{i, 2}\right)
$$

$\pi\left(y_{i, 2}>y_{j, 2}\right)$ denotes the probability that $i$ 's output in stage two is greater than the output of agent $j$. With our assumptions regarding the production function and random terms, this expression can be rewritten as follows:

$$
E U_{i}\left(w_{h i g h}, w_{m e d}, e_{i, 2}, e_{j, 2}\right)=F_{\epsilon_{j, 2}-\epsilon_{i, 2}}\left[e_{i, 2}-e_{j, 2}\right]\left(w_{h i g h}-w_{m e d}\right)+w_{m e d}-C\left(e_{i, 1}, e_{i, 2}\right)
$$

\footnotetext{
${ }^{3}$ The parameters chosen in our experimental treatments (see below) ensure that all participation constraints are fulfilled. We therefore do not explicitly consider them here.
} 
where $F_{\epsilon_{j, 2}-\epsilon_{i, 2}}[\cdot]$ denotes the cdf of the difference between random terms $\epsilon_{j, 2}, \epsilon_{i, 2}$. Maximizing $E U_{i}(\cdot)$ over $e_{i, 2}$ yields the following first-order condition:

$$
f_{\epsilon_{j, 2}-\epsilon_{i, 2}}\left(e_{i, 2}-e_{j, 2}\right)\left(w_{\text {high }}-w_{\text {med }}\right)=\frac{\partial C(\cdot)}{\partial e_{i, 2}}
$$

Assuming symmetry yields $f_{\epsilon_{j, k}-\epsilon_{i, k}}(0)=\frac{1}{2 q}$ for $\epsilon_{i, k}, \epsilon_{j, k} \sim U[-q, q]$. The symmetric subgame perfect Nash equilibrium of the two-stage tournament thus entails the following second-stage effort level $e_{i, 2}^{*, T S}$ for the two agents who participate in the final:

$$
e_{i, 2}^{*, T S}=\frac{\left(w_{\text {high }}-w_{\text {med }}\right) c}{4 q}
$$

Given that both finalists play this equilibrium, the expected utility gain in the final, i.e., the continuation value for an agent in the first stage is given as follows

$$
E V_{i, 2}=w_{\text {med }}+\frac{1}{2}\left[w_{\text {high }}-w_{\text {med }}\right]-\frac{\left(e_{i, 2}^{*, T S}\right)^{2}}{c}
$$

An agent who reaches the final earns a wage of $w_{\text {med }}$ for sure. By exerting stage-two effort $e_{i, 2}^{*, T S}$, he has the chance to receive the higher wage $w_{\text {high }}$ instead. In the symmetric equilibrium, this occurs with probability $\frac{1}{2}$. Moreover, he has to pay the cost of effort exertion in the second stage.

Turning to the analysis of the tournament's first stage, it is obvious that (in expected values) this stage can be modelled as a one-stage tournament between four agents with two winner prizes $E V_{i, 2}$ and two loser prizes $w_{\text {low }}$ (see Rosen 1986). The derivation of equilibrium effort for such a tournament follows the same steps as above. Alternatively we can apply a result from Orrison et al. (2004) who show that equilibria of fully symmetric one-stage tournaments are not affected by "organizational replication" for our specification of the production function, cost function, and random terms. This implies that an equilibrium in a tournament with two identical participants and one winner prize is also an equilibrium in a tournament with four identical participants and two winner prizes. ${ }^{4}$

\footnotetext{
${ }^{4}$ More generally Orrison et al. (2004) show that an equilibrium in a tournament with $n$ participants and $\frac{1}{2} n$ winner prizes is still an equilibrium in a tournament with $m n$ participants and $\frac{m}{2} n$ winner prizes.
} 
As a shortcut we can therefore use the solution for $e_{i, 2}^{*, T S}$ and simply replace $w_{\text {high }}$ and $w_{\text {med }}$ with $E V_{i, 2}$ and $w_{\text {low }}$ to obtain the equilibrium effort level for the first stage:

$$
e_{i, 1}^{*, T S}=\frac{\left(E V_{i, 2}-w_{\text {low }}\right) c}{4 q}=\frac{\left(w_{\text {med }}-w_{\text {low }}+\frac{1}{2}\left[w_{\text {high }}-w_{\text {med }}\right]-\frac{\left(e_{i, 2}^{*, T S}\right)^{2}}{c}\right) c}{4 q}
$$

This expression illustrates the two components of incentive provision in multi-stage tournaments. By winning the first stage and qualifying for the final, an agent receives an immediate wage gain $\left(w_{\text {med }}-w_{\text {low }}\right)$, but additionally has the option to compete in the final and win the top prize $w_{\text {high }}$. The value of this option is

$$
\frac{1}{2}\left[w_{\text {high }}-w_{\text {med }}\right]-\frac{\left(e_{i, 2}^{*, T S}\right)^{2}}{c}
$$

Several aspects of the model deserve special emphasis. First, our design closely follows the original model of elimination tournaments by Rosen (1986) with one notable exception: instead of having two semifinals with two participants each, who compete for one slot in the final, we analyze a setup with four participants competing for two slots in the final. While both variants are theoretically equivalent for symmetric agents, we employ the latter because it allows us to design a one-stage tournament which is procedurally as close as possible to our main treatment. ${ }^{5}$ Note also that we abstract from heterogeneous abilities of participants in order to keep the design as simple and parsimonious as possible. This allows us to focus on the incentive aspect of elimination tournaments by ruling out selection of more able individuals into higher positions of a hierarchy. Including the selection aspect of tournaments would be an interesting follow-up to our study.

\footnotetext{
${ }^{5}$ In particular - as will become clear in the next section — both tournaments have the same number of participants and subjects compete for the same number of promotions.
} 


\section{Experimental design}

\subsection{Treatments and hypotheses}

Our experiment comprises of three treatments that allow us to study behavior in multistage tournaments from different angles. For all treatments our benchmark is the prediction of the symmetric subgame perfect Nash equilibrium. The main treatment $T S$ is a two-stage elimination tournament with four participants competing for $w_{h i g h}$, $w_{\text {med }}$, and $w_{\text {low }}$ as discussed in the previous section. We compare this treatment to a one-stage tournament $(O S)$ in which four subjects compete for two top positions. The two subjects with the highest output levels receive a wage $w_{\text {med }}^{\prime}$ in $O S$ while the two losers of the competition receive a wage $w_{l o w}$. The $O S$ treatment fulfills several purposes. First, it serves as a validity check for our results given that a number of studies on one-stage tournaments already exists. In particular, our parametrization of this treatment is very close to a treatment from Orrison et al. (2004).

More importantly, however, the $O S$ treatment allows us to investigate whether onestage tournaments are behaviorally different from multi-stage ones. To investigate this question we design $O S$ such that it is strategically equivalent to the first stage of the twostage tournament TS. As discussed in the previous section the first stage of a two-stage tournament can be interpreted as a one-stage tournament in which agents compete for the expected value of participating in the second stage. Strategic equivalence between $O S$ and the first stage of $T S$ is thus achieved by keeping $w_{\text {low }}$ constant and choosing:

$$
w_{\text {med }}^{\prime}=E V_{i, 2}=w_{\text {med }}+\frac{1}{2}\left[w_{\text {high }}-w_{\text {med }}\right]-\frac{\left(e_{i, 2}^{*, T S}\right)^{2}}{c}
$$

In other words, the wage for the promoted agents in the one-stage tournament $\left(w_{\text {med }}^{\prime}\right)$ is equivalent to the wage $w_{\text {med }}$ from TS plus the option value of participating in the final of $T S$. This choice implies that equilibrium effort levels in the $O S$ treatment and in the first stage of the $T S$ treatment are the same. We can therefore formulate 
the behavioral equivalence hypothesis:

$$
e_{i, 1}^{O S}=e_{i, 1}^{T S}
$$

In addition to comparing behavior in tournaments with different numbers of stages, we are interested in how a change in the wage structure influences behavior in multistage tournaments. This comparative statics exercise is of practical interest. Wages are one of the variables which (within certain bounds) are most amenable to manipulation in organizational design. We therefore compare our main treatment to a second twostage tournament with a more $\underline{\text { convex }}$ wage profile $(T S \underline{C})$. It is identical to the TS treatment with the exception that the intermediate wage $w_{m e d}^{\prime \prime}$ in this treatment is smaller than in TS. This implies weaker incentives (lower equilibrium effort) in the first stage and a higher wage spread and higher equilibrium effort in the second stage (wage structure hypothesis):

$$
\begin{aligned}
& e_{i, 1}^{T S C}<e_{i, 1}^{T S} \\
& e_{i, 2}^{T S C}>e_{i, 2}^{T S}
\end{aligned}
$$

Finally, we analyze behavior in our main treatment across stages. In the TS treatment wages are chosen such that equilibrium efforts in the first and second stage are equal. Remember that the two elements of incentives in multi-stage tournaments are the wage spread and the option value of competing for further promotions. In the final stage, the option value is zero because there are no further promotions beyond that stage. To make the tournament in the TS treatment incentive maintaining in the sense of Rosen (1986), this decrease in the option value in the second stage is offset by an appropriate increase in the wage spread $\left(w_{\text {high }}-w_{\text {med }}>w_{\text {med }}-w_{\text {low }}\right)$. Comparing behavior across stages thus allows to test the incentive maintenance hypothesis:

$$
e_{i, 1}^{T S}=e_{i, 2}^{T S}
$$

Experimental parameters and the resulting equilibrium efforts for all treatments are shown in Table 1. When deciding on their efforts, subjects could choose any integer 
$e_{i, k} \in\{0,1, \ldots, 125\}$. The effort costs in each stage are given by

$$
C\left(e_{i, k}\right)=\frac{e_{i, k}^{2}}{2250}
$$

and $\epsilon_{i, k} \sim U[-60,60] .{ }^{6}$ The parameters chosen imply equilibrium efforts of 74 in both stages of $T S$ and in $O S$, the lower intermediate wage in $T S C$ changes equilibrium efforts to 42 in the first stage and 100 in the second stage of this treatment.

\begin{tabular}{lccc}
\hline \hline Treatment & OS & TS & TSC \\
\hline$c$ & 2250 & 2250 & 2250 \\
$q$ & 60 & 60 & 60 \\
$w_{\text {high }}$ & - & 20 & 20 \\
$w_{\text {med }}^{\prime} / w_{\text {med }} / w_{\text {med }}^{\prime \prime}$ & 13.62 & 12.11 & 9.33 \\
$w_{\text {low }}$ & 5.73 & 5.73 & 5.73 \\
\hline$e_{i, 1}^{*}$ & 74 & 74 & 42 \\
$e_{i, 2}^{*}$ & - & 74 & 100 \\
\hline \hline
\end{tabular}

Table 1: Experimental parameters and resulting equilibrium predictions.

\subsection{Experimental procedures}

The experiment was conducted at the BonnEconLab of the University of Bonn. A total of 96 subjects in six sessions were divided into groups of four. We employed a one-shot between-subjects design, i.e., subjects participated either in the $O S$, the $T S$ or the TSC treatment. The experiment was programmed and conducted with the software z-Tree (Fischbacher 2007); subjects were recruited using the online recruitment system by Greiner (2003).

Before the tournament started, subjects received detailed written instructions on the respective treatment they took part in $(O S, T S$ or $T S C)$. These were neutrally framed

\footnotetext{
${ }^{6}$ Note that, while in equilibrium all players make positive profits, the range of feasible efforts and the specification of the cost function imply that in principle subjects could make losses. In the few cases where this occurred, losses were deducted from the showup-fee.
} 
and did not contain potentially value-laden terms like "tournament", "final", "winner", etc. After reading the instructions subjects completed several control questions. The experiment started only after all participants had answered all control questions correctly. During the tournament, subjects simultaneously entered their effort decision and were then asked to state their expectations about the other participants' efforts on the next screen. This question was not announced beforehand. After the first stage participants in $T S$ and $T S C$ were only informed about the realization of their own random draw and about whether they had been promoted to the second stage. The finalists then again made an effort choice and entered their expectation about their opponent's effort. At the end of the tournament subjects in all treatments were informed about their earnings and asked to fill in a questionnaire. The structure of the experimental session ensured that subjects' decisions in the tournament can be treated as independent observations. The whole experimental session lasted on average 100 minutes and subjects earned an average of 18.25 Euro (1 Euro $=1.26$ USD at the time of experiment), including a showup-fee of 4 Euro and a fixed payment of 3 Euro for completing the questionnaire.

Note that our experimental procedures differ from previous tournament experiments in that we implement a one-shot interaction structure. Previous experiments have typically used repeated interactions. The advantage of the latter is that it allows for learning, which is potentially important given the non-trivial decision environment in tournaments. A potential downside, however, is that repeated game structures question the validity of static equilibrium predictions. Since we are explicitly interested in testing theoretical predictions, we decided to use a one-shot design. This has the additional advantage that stakes in the one-shot interaction are relatively high. We check the regularity of our results by comparing the outcome of our $O S$ treatment with those of similar repeated tournaments. Finding similar results would make us confident that our main findings are not driven by the one-shot character of our set-up, but instead by treatment differences. 


\section{Results}

In this section we first test whether the results for the one-stage tournament replicate earlier findings from similar tournaments. We then study the dynamic aspect of multistage tournaments by comparing the one-stage tournament $O S$ to the strategically equivalent first stage of the two-stage tournament $T S$. In a third step, we investigate the effects of differences in the wage structure on effort provision by comparing the two-stage tournaments $T S$ and TSC. Finally, we address the question whether the TS treatment is incentive maintaining by analyzing behavior in the first and second stage of the tournament.

\subsection{Behavior in the one-stage tournament}

Table 2 reports effort decisions in the $O S$ treatment (column 1). Two points are worth noting. First, efforts are on average very close to the theoretical predictions. While the average effort of 69.9 is slightly below the Nash prediction of 74, median effort coincides exactly with the predicted effort level. Second, there is substantial heterogeneity in subjects' behavior (see also Figure 1).

Both observations are in line with previous findings from symmetric one-stage designs (e.g., Bull et al. 1987, Eriksson et al. 2006). In particular, our results replicate those found by Orrison et al. (2004) who observe an average effort of 73.3 for an almost identical tournament which was repeated 20 times using lower stakes. The similarity of our results to those of Orrison et al. (2004) show that one of the most important findings in the experimental literature on symmetric promotion tournaments - average effort being close to Nash predictions - is quite robust with respect to using one-shot vs. repeated interactions and with respect to increased stake sizes.

Result 1: Average behavior in the one-stage tournament is close to the predictions of the symmetric Nash equilibrium. 


\begin{tabular}{lccc}
\hline \hline Treatment & OS & TS & TSC \\
\hline Average Effort & 69.9 & 89.2 & 82.4 \\
Median Effort & 74 & 91 & 83 \\
$\min$ (Effort) & 1 & 40 & 40 \\
$\max$ (Effort) & 125 & 125 & 125 \\
Variance & 913.3 & 359.7 & 605.4 \\
\hline$e^{*}$ & 74 & 74 & 42 \\
\hline \hline
\end{tabular}

Table 2: First stage behavior in the OS, TS, and TSC treatment.

\subsection{Testing behavioral equivalence}

Our one-stage tournament and the first stage of the two-stage tournament TS are strategically equivalent in the sense that the wage $w_{\text {med }}^{\prime}$ in the one-stage tournament includes the equilibrium option value of participating in the second stage of the two-stage tournament. A comparison of $e_{i, 1}^{O S}$ and $e_{i, 1}^{T S}$ therefore serves as a test of how subjects in the two-stage tournament perceive this option value. If subjects, for instance, do not take the option value into account when deciding on their first stage effort in $T S$, efforts in this stage should be lower compared to the $O S$ treatment. If subjects evaluate the option value correctly, efforts in both treatments should be identical. Columns 1 and 2 of Table 2 show that behavior differs strongly between the two treatments. Average effort in the first stage of the TS treatment is 89.2, while median effort is 91 . Thus, subjects behave much more competitively in the multi-stage tournament, exerting efforts which are more than $20 \%$ higher than those of their counterparts in the $O S$ treatment. A Mann-Whitney U-test confirms that this treatment difference is highly significant $(p=0.005$, two-sided $)$.

Comparing effort levels in the two treatments to the theoretical predictions derived in section 3 indicates that it is excess effort in TS rather than "too low" effort in $O S$ that drives the treatment difference. A t-test with the null hypothesis that efforts are equal to Nash predictions confirms this: the null hypothesis is rejected in the TS 
treatment $(p<0.001)$, but cannot be rejected in $O S(p=0.453){ }^{7}$ Subjects' effort choices suggest that they are not naive in the sense that they ignore the second stage. Quite to the contrary, the two-stage elimination tournament seems to trigger especially competitive behavior in the first stage.

The treatment difference is not just driven by some subjects choosing extreme effort levels in the TS treatment. A closer look at the distributions of first stage efforts in Figure 1 reveals instead that the whole effort distribution is shifted to the right in the $T S$ treatment. As a consequence, efforts are less dispersed (Levene's test for equality of variances, $p<0.05$, two-sided). The effort distribution illustrates that exerting excess effort is quite widespread in $T S: 84 \%$ of subjects choose efforts higher than the equilibrium effort level of 74 . This compares to only $47 \%$ in the $O S$ treatment. The strong difference between treatments is also reflected at the lower tail of the distribution. While the lowest effort in the $O S$ treatment is 1 , no subject exerts effort below 40 in the TS treatment.

Result 2: Efforts in the first round of TS are significantly higher than in the OS treatment. This difference is driven by excess effort in TS.

\subsection{Wage structures in two-stage tournaments}

In our next treatment comparison we investigate the behavioral effects of different wage structures in two-stage tournaments. Our main interest concerns the question whether first stage excess effort is a robust phenomenon. Is excess effort in the first stage an artefact of the specific wage structure we used in the TS treatment, e.g., is it specific

\footnotetext{
${ }^{7}$ Note that the derivation of equilibrium predictions is based on the assumption of risk neutrality. We check the validity of this assumption by eliciting subjects' risk preferences with an incentive compatible lottery procedure. It turns out that our experimental subject pool is close to risk neutrality: the median subject in all three treatments is risk neutral, and the certainty equivalent of more than $50 \%$ of subjects lies in a range of $+/-0.25$ Euro around the risk neutral certainty equivalent (equal to 2 Euro for the lottery that was used). In addition, effort levels in $T S$ are above theoretical predictions irrespective of the subjects' degree of risk aversion. Thus excess effort is not driven by subjects' risk preferences.
} 


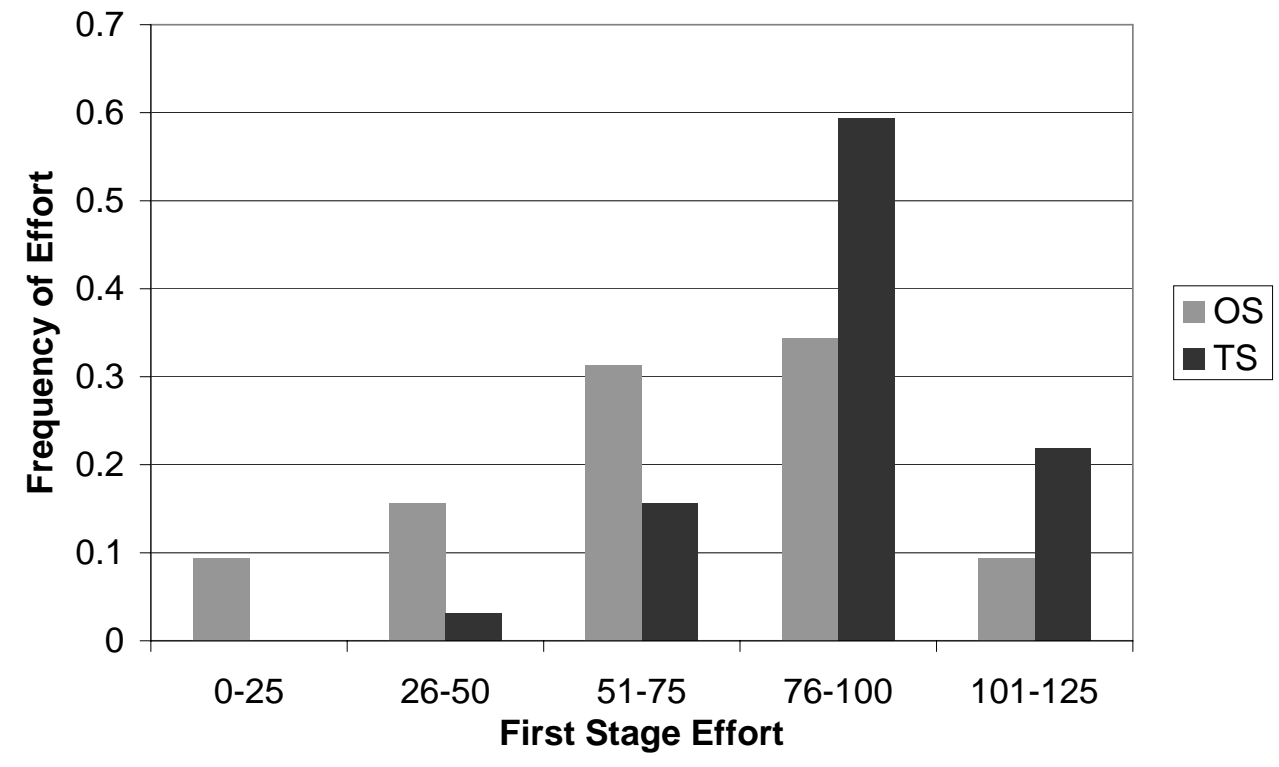

Figure 1: Frequency of effort choices in the OS treatment and the first stage of the TS treatment.

to the incentive maintaining wage spreads? Or do we observe excess effort even if we reduce the incentive to provide effort in the first stage? To analyze these questions we implemented the TSC treatment, which is identical to the TS treatment except that the subject who produces less output in the second stage receives only $w_{m e d}^{\prime \prime}=9.33$ Euro instead of $w_{\text {med }}=12.11$ Euro. This more convex wage profile has the following theoretical implications. Incentives to provide effort in the first stage are weakenedthe equilibrium effort level in the first stage of $T S C$ is only 42 instead of 74 in the TS treatment. In the second stage, equilibrium effort increases from 74 to 100 (see Table $1)$.

Column 3 of Table 2 summarizes behavior in the first stage of the TSC treatment. Efforts in the first stage are much higher than theoretically predicted. The average effort level is 82.4 points, about 40 points higher than the equilibrium effort level of 42 . A ttest with the null hypothesis that efforts are equal to the Nash prediction confirms that effort choices are significantly above the equilibrium prediction $(p<0.001)$. Indeed, about $88 \%$ of subjects choose efforts higher than 42 . This suggests that excess effort 
in the first stage of our two-stage tournaments is robust to differences in the wage structure. Comparing effort choices in the TSC treatment to those observed in TS reveals that subjects react only weakly to the change in the wage structure between the two treatments. Average effort in TSC is only 7 points lower than in TS. This finding is striking given that there is a 32-point difference in equilibrium efforts. Despite the fact that first stage incentives are much weaker than in $T S$, effort choices do not differ significantly between the two treatments (Mann-Whitney U-test, $p=0.245$, two-sided).

Result 3: The more convex wage structure in TSC induces even higher excess effort in the first stage. First stage efforts in TS and TSC are not significantly different, despite much weaker incentives in the TSC treatment.

\subsection{Testing incentive maintenance}

Finally, we turn to behavior in the second stage of the $T S$ treatment. Remember that parameters were chosen such that the tournament in TS is incentive maintaining, i.e., equilibrium effort levels are the same (equal to 74) in both stages. We know already that efforts are above the equilibrium prediction in the first stage of $T S$. In this sense, we can reject the hypothesis of equilibrium effort choices in both stage of TS. It remains to show, however, whether effort levels are the same in both stages of the tournament, or whether they differ. Does the two-stage character of $T S$ induce above equilibrium effort also in the second stage or do players reduce efforts relative to their first stage behavior?

In turns out that the the evidence is mixed. On average, effort decreases when comparing behavior across stages in the TS treatment. Average effort in the first stage is 89 , in the final it goes down to 82 . The median effort choice decreases from 91 to 87.5. Note that average effort in the first stage includes the efforts of those who did not make it to the second stage. Since - by design of the promotion tournament - the latter usually exerted lower effort, the decrease from stage one to stage two is larger if we 
consider only finalists' behavior. Their average effort in the first stage is 96, implying that on average finalists decrease their effort by 14 points. However, these numbers hide considerable heterogeneity on an individual level: the fraction of finalists who decrease their effort in the second stage is only slightly higher than the fraction of subjects who increase it (50\% and 44\%, respectively). It is therefore not surprising that the overall decrease in finalists' efforts is insignificant (Wilcoxon signed rank test, p-value $=0.289$ ) However, those who adjust their effort downwards on average do so much stronger than those who raise their effort.

Does this mean that excess effort in the first stage just mirrors the lower efforts in the second stage? That is, do subjects expect low effort levels and thus low effort costs in the second stage and therefore increase their first stage effort due to a higher (perceived) option value? While this might be the case for some finalists, we can rule out that it accounts for first stage behavior on a more general level as, on average, effort choices in the second stage are still above the equilibrium prediction. We can also use the observed first stage effort choice of a subject to calculate the option value implicitly underlying her decision. Using this option value, we can then construct the (hypothetical) second stage effort level which would rationalize the first stage effort choice of the subject at hand. For instance, if a subject beliefs that all players will exert zero effort in the second stage, her subjective option value increases to $\frac{1}{2}\left[w_{\text {high }}-w_{\text {med }}\right]$ since $\frac{\left(e_{i, 2}^{T S}\right)^{2}}{c}=0$ (see section 2 ). Assuming this option value instead of the equilibrium option value rationalizes a first stage effort of 97 (using the wages and cost parameters of $T S)$. Conducting the calculation for the first stage effort choice of the median subject in TS (equal to 91) yields an implied second stage effort of 37. I.e., if the median subject had expected a second stage effort level of 37, the perceived option value would rationalize her observed first stage effort choice. This value is, however, far below the actual effort choices in the second stage (cp. Table 2). ${ }^{8}$ The expectation of low second

\footnotetext{
${ }^{8}$ The same exercise for the TSC treatment yields a value of 14 while the average effort level actually observed in the second stage of this treatment is 82.9. Thus, although subjects on average choose efforts below the equilibrium value of 100 in the second stage of $T S C$, this cannot explain the excess efforts
} 
stage effort levels can thus not account for the observed excess effort in the first stage of $T S$.

Result 4: Efforts in the second stage of TS are lower, but not significantly different from efforts in the first stage. In this sense, the TS treatment is incentive maintaining.

\section{Concluding remarks}

Promotions in most hierarchical organizations take the form of multi-stage elimination tournaments. In this paper we have studied behavior in such tournaments with simple laboratory experiments. Our results demonstrate the importance of carefully analyzing the incentive effects of promotions in multi-level hierarchies. They show that the basic logic of incentive provision in multi-stage elimination tournaments works in the sense that people take future promotion possibilities into account when deciding on current work effort. However, we also observe important departures from theoretical predictions. Subjects tend to exert excess effort in the first stage of our two-stage elimination tournament. By contrast, we do not observe this phenomenon in a strategically equivalent one-stage tournament. Under a more convex wage structure, the overprovision of effort is even more pronounced.

Our experiments suggest that behavior in multi-stage tournaments deviates from behavior in one-stage tournaments in a systematic way. Our data do, however, not allow us to give a definite answer on the precise mechanism that causes this change in behavior. Several factors may act in concert: it could be that subjects experience additional non-monetary "joy of winning" when being promoted (Parco et al. 2005), which might be more pronounced when the hierarchy has more layers. An additional potential rationale for subjects' behavior are preferences for status (Moldovanu et al. 2007). Multi-stage tournaments with their more precise definition of hierarchical level exerted in the first stage. 
(and status) might trigger especially competitive behavior of status concerned agents. Our data on subjects' expectations are consistent with these interpretations: about two thirds of subjects choose an effort above the second-highest effort level that they expect from their competitors, irrespective of the absolute level of the effort expectation.

The observed behavior could also help to rationalize why firms rely so strongly on promotions as incentive device, even in work environments where more direct performance pay is feasible. Excess effort in early stages of multi-stage tournaments makes this form of incentive provision comparatively "cheap" for the principal as it decreases the wage cost per unit of effort. For instance, in our TS tournament this cost is 9.81 Cent in theory, but only 8.36 Cent in practice. This implies that a principal who implemented the TS wage scheme had to pay less for every unit of effort (and production) than theoretically predicted. Which wage profile a principal or tournament designer actually prefers depends on his objectives. In some situations it might be sufficient to concentrate on the cost per effort. In other situations a tournament designer may, for example, put special emphasis on the performance of agents in higher stages of the tournament. The TSC treatment suggests that excess effort in early stages eventually might come at the cost of reduced performance in later stages if the wage structure becomes too convex. Independent of the specific objective function, a tournament designer should take into account that agents' behavior can ultimately change the optimal architecture of promotion tournaments in terms of wage profiles, promotion rates, etc. 


\section{References}

Baker, George, Michael Gibbs and Bengt Holmström (1994): “The Internal Economics of the Firm: Evidence from Personnel Data." The Quarterly Journal of Economics, 109:881-919.

Belzil, Christian and Michael Bognanno (2008): "Promotions, Demotions, Halo Effects, and the Earnings Dynamics of American Executives." Journal of Labor Economics, 26:287-310.

Bognanno, Michael L. (2001): "Corporate Tournaments." Journal of Labor Economics, 19:290-315.

Bull, Clive, Andrew Schotter and Keith Weigelt (1987): "Tournaments and Piece Rates: An Experimental Study." Journal of Political Economy, 95:1-33.

Dohmen, Thomas and Armin Falk (2006): "Performance Pay and Multi-dimensional Sorting: Productivity, Preferences and Gender." IZA Discussion Paper, 2001.

Ehrenberg, Ronald G. and Michael L. Bognanno (1990): "Do Tournaments Have Incentive Effects?" Journal of Political Economy, 98:1307-1324.

Eriksson, Tor (1999): "Executive Compensation and Tournament Theory: Empirical Tests on Danish Data." Journal of Labor Economics, 17:262-280.

Eriksson, Tor, Sabrina Teyssier and Marie-Claire Villeval (2006): "Self-Selection and the Efficiency of Tournaments." IZA Discussion Paper, 1983.

Falk, Armin, Ernst Fehr and David Huffman (2008): "The Power and Limits of Tournament Incentives." Working Paper, University of Bonn.

Fischbacher, Urs (2007): "z-Tree - Zurich Toolbox for Ready-made Economic Experiments." Experimental Economics, 10:171-178. 
Greiner, Ben (2003): “An Online Recruitment System for Economic Experiments." In Kremer, Kurt and Volker Macho, editors, Forschung und wissenschaftliches Rechnen 2003. GWDG Bericht 63, pages 79-93. Ges. für Wiss. Datenverarbeitung, Göttingen.

Harbring, Christine and Bernd Irlenbusch (2003): "An Experimental Study on Tournament Design." Labour Economics, 10:443-464.

Harbring, Christine and Bernd Irlenbusch (2008): "How Many Winners Are Good to Have? On Tournaments with Sabotage." Journal of Economic Behavior and Organization, 65:682-702.

Knoeber, Charles R. and Walter N. Thurman (1994): "Testing the Theory of Tournaments: An Empirical Analysis of Broiler Production." Journal of Labor Economics, 12:155-179.

Lazear, Edward P. (2000): "Performance Pay and Productivity." American Economic Review, 90:1346-1361.

Lazear, Edward P. and Michael Gibbs (2008): Personnel Economics in Practice. John Wiley \& Sons, 2nd edition.

Lazear, Edward P. and Sherwin Rosen (1981): "Rank-Order Tournaments as Optimum Labor Contracts." Journal of Political Economy, 89:841-864.

Moldovanu, Benny and Aner Sela (2006): "Contest Architecture." Journal of Economic Theory, 126:70-96.

Moldovanu, Benny, Aner Sela and Xianwen Shi (2007): "Contests for Status." Journal of Political Economy, 115:338-363.

Orrison, Alannah, Andrew Schotter and Keith Weigelt (2004): "Multiperson Tournaments: An Experimental Investigation." Management Science, 50:268-279. 
Parco, James E., Amnon Rapoport and Wilfried Amaldoss (2005): "Two-stage Contests with Budget Constraints: an Experimental Study." Journal of Mathematical Psychology, 49:320-338.

Rosen, Sherwin (1986): "Prizes and Incentives in Elimination Tournaments." American Economic Review, 76:701-715.

Schotter, Andrew and Keith Weigelt (1992): "Asymmetric Tournaments, Equal Opportunity Laws, and Affirmative Action: Some Experimental Results." The Quarterly Journal of Economics, 107:511-539. 